\title{
L'assorbimento del glucosio in dialisi peritoneale: strumenti utili per il team multidisciplinare
}

\author{
Anna Laura Fantuzzi ${ }^{1}$, Elisa Berri ${ }^{2}$, Lida Tartaglione ${ }^{3,4}$, Rossella Giannini $^{5}$, Sara Dominjanni ${ }^{6}$, Silvia Porreca $^{7}$ \\ ${ }^{1}$ Dietista già Coordinatore Nutrizione e Dietetica Aziendale, AUSL Modena, Comitato Scientifico ASAND (Associazione Scientifica \\ Alimentazione Nutrizione e Dietetica) - Italy \\ ${ }^{2}$ Dietista, Nutrizione e Dietetica Aziendale, AUSL Modena - Italy \\ ${ }^{3}$ Dirigente Medico, UOC Nefrologia e Dialisi, ASL RM5 - P.O. Tivoli (RM) - Italy \\ ${ }^{4}$ Dirigente Medico, Dipartimento Medicina Traslazione e di Precisione, Sapienza Università di Roma, Roma - Italy \\ ${ }^{5}$ Dietista, SSD Malattie del Metabolismo e Nutrizione Clinica, Azienda Ospedaliero Universitaria Policlinico di Modena, Modena - Italy \\ ${ }^{6}$ Dirigente Medico, UOC Nefrologia e Dialisi Ospedale Sant'Eugenio - ASL Roma 2, Roma - Italy \\ 7Dirigente Medico, UOC. di Nefrologia e Dialisi, P.O. "Di Venere" Bari - ASL BA, Segretario del Gruppo di Progetto di Dialisi Peritoneale \\ della Società Italiana di Nefrologia - Italy
}

\begin{abstract}
Glucose absorption in peritoneal dialysis: useful tools for a multidisciplinary team
Dialysis patients are often affected by protein energy wasting and the maintenance of an optimal nutritional state is a difficult goal to achieve. Moreover protein energy wasting is one of the strongest risk factors for mortality in chronic dialysis patients.

To estimate glucose absorption in peritoneal dialysis is essential to determine patient's dietary energy requirements and to prevent possible metabolic complications.

The currently accepted methods of estimating glucose absorption are two. The first one is based on the average glucose absorption of continuous ambulatory peritoneal dialysis (CAPD) and is calculated with the Grodstein et al. formula. The second one is based on the Peritoneal equilibration test curves (D/DO formula, Bodnar et al.) and takes into account transport characteristics. None of the two formulas perfectly calculated the absolute glucose absorption, even if the D/DO is much closer to the true value, compared to Grodstein et al. formula. In this paper we described the multidisciplinary management experience of peritoneal dialysis patients, with a focus on the estimation of the patient's energy requirements. Analyzing glucose absorption in a systematic way and monitoring the changes in glucose absorption during the time could significantly contribute to adjusting nutritional treatment. However it is always necessary to critically evaluate the results obtained by both formulas.
\end{abstract}

Keywords: Glucose, Glucose absorption, Peritoneal dialysis

\section{Introduzione}

La stima dell'assorbimento del glucosio è importante per valutare il fabbisogno energetico e controllare le possibili alterazioni metaboliche in corso di dialisi peritoneale (DP) per ogni paziente. I due metodi presi in considerazione, Grodstein et al. e D/DO formula, dal punto di vista pratico, sono entrambi utilizzabili anche se ciascuno con dei limiti. In casi particolari di pazienti malnutriti con alterazioni metaboliche rilevanti

Received: January 5, 2022

Accepted: January 10, 2022

Published online: February 8, 2022

Indirizzo per la corrispondenza:

Anna Laura Fantuzzi

Via Bismantova 4

42019 Scandiano (RE) - Italy

annalaurafantuzzi@gmail.com e di difficile correzione è possibile considerare la possibilità di calcolare l'assorbimento reale di glucosio giornaliero. La Grodstein et al. formula può essere utilizzata per i pazienti in trattamento con Continous Ambulatorial Peritoneal Dialysis (CAPD), mentre non può essere utilizzata nei pazienti in Automated Peritoneal Dialysis (APD). Questa formula non tiene in considerazione le caratteristiche di trasporto della membrana peritoneale e quindi risulta correlata a una maggiore possibilità di errore nei soggetti classificati come alti o bassi trasportatori. Con l'introduzione del PET in DP è nata la possibilità di valutare l'assorbimento del glucosio in base alle caratteriste di trasporto del singolo paziente. La D/DO formula permette di stabilire l'assorbimento di glucosio in modo individualizzato e, potendo calcolare questa grandezza in diversi periodi di tempo, può essere utilizzata sia in CAPD che in APD. La D/ D0 formula ha però il limite di essere il risultato di una metodica semiquantitativa che fornisce valori relativi e può essere soggetta a errori nella procedura di esecuzione del test che 
potrebbero alterare i dati utilizzati nel calcolo. I risultati ottenuti da entrambe queste formule possono essere valutati singolarmente oppure essere messi a confronto; non essendo però né valori assoluti, né costanti nel tempo e né sufficienti, da soli, a definire le necessità caloriche del paziente, è necessario che siano sempre valutati in modo critico. In questo articolo si riporta l'esperienza della gestione multidisciplinare dei pazienti in trattamento dialitico peritoneale, presso l'Unità Operativa di DP dell'Azienda Ospedaliero-Universitaria Policlinico di Modena oggetto di una tesi di Master in Dietistica Renale dell'Università di Modena e Reggio Emilia nell'anno accademico 2011-2012. La corretta valutazione dell'assorbimento di glucosio è un ulteriore elemento importante nel percorso di definizione multidisciplinare del trattamento adeguato al raggiungimento degli obiettivi del piano terapeutico, incluso il trattamento nutrizionale del paziente in DP.

\section{La dialisi peritoneale}

La dialisi peritoneale è una metodica che consta di una o più fasi definite "scambio» e che può essere suddivisa in una fase di carico (il liquido di dialisi entra nella cavità peritoneale attraverso il catetere peritoneale), in una fase di sosta (depurazione effettiva) e in una fase di scarico (il liquido di dialisi saturo viene drenato e poi sostituto con del nuovo liquido).

Nella fase di sosta, una volta introdotto il liquido di dialisi e messi a contatto i due compartimenti attraverso il peritoneo, $\mathrm{i}$ meccanismi chimico-fisici di diffusione (secondo un gradiente di concentrazione), ultrafiltrazione (secondo un gradiente di pressione osmotica, oncotica e idrostatica) e convezione (per trascinamento assieme al solvente, proporzionale all'ultrafiltrazione) consentono la rimozione dei cataboliti uremici (urea, creatinina, acido urico, ecc.), la rimozione dei fluidi corporei in eccesso, il controllo degli elettroliti (fosforo, potassio, ecc.) e la correzione dell'equilibrio acido-base. II liquido di dialisi è quindi il principale strumento per la rimozione dell'acqua e delle tossine, per fornire sostanze utili e bilanciare gli squilibri idroelettrolitici del paziente uremico.

\section{II liquido di dialisi}

Il liquido di dialisi è una miscela sterile, a composizione nota, contenente principalmente elettroliti (calcio, cloro, sodio, magnesio e potassio), glucosio e lattato (o bicarbonato). Attualmente il glucosio è l'agente osmotico maggiormente utilizzato nelle soluzioni per DP. La sua concentrazione nel liquido di dialisi varia dall' $1,36 \%$ al $4,25 \%$. In base alla concentrazione di glucosio le soluzioni possono essere classificate in: - Isotonica: 1,36\% (destrosio anidro)-1,5\% (destrosio monoidrato); - Mediamente ipertonica: 2,27\% (destrosio anidro)-2,5\% (destrosio monoidrato); - Ipertonica: $3,86 \%$ (destrosio anidro)-4,25\% (destrosio monoidrato). Dal momento che il glucosio è una molecola a basso peso molecolare (P.M. = $180 \mathrm{D})$, attraversa facilmente la membrana peritoneale e viene richiamato nel circolo ematico durante la fase di sosta. II riassorbimento dipende dalla sua concentrazione nella soluzione infusa, dal tempo di sosta e dalla permeabilità individuale della membrana peritoneale. Negli ultimi anni hanno fatto la loro comparsa miscele che, al posto del glucosio come agente osmotico, contengono amminoacidi o polimeri del glucosio (p. es., icodestrina al 7,5\%). Negli schemi di dialisi peritoneale, quando vengono impiegate, non sostituiscono totalmente le sacche del trattamento ma possono essere impiegate in un singolo scambio nelle 24 ore come parte integrante in un trattamento di CAPD o APD (1).

\section{Gli schemi di dialisi}

Lo schema di dialisi utilizzato nella CAPD fornisce le seguenti indicazioni terapeutiche: numero di scambi, orario degli scambi, tipo di sacche utilizzate in ogni singolo scambio (glucosio all'1,36\%-1,5\% ecc., icodestrina al 7,5\%, ecc.), quantità di liquido da scambiare, bilancio idrico (entrate, ultrafiltrato, diuresi), peso del paziente e pressione arteriosa (Fig. 1). Al variare delle necessità cliniche lo schema può subire modifiche in termini di: tipo di formula (glucosio,

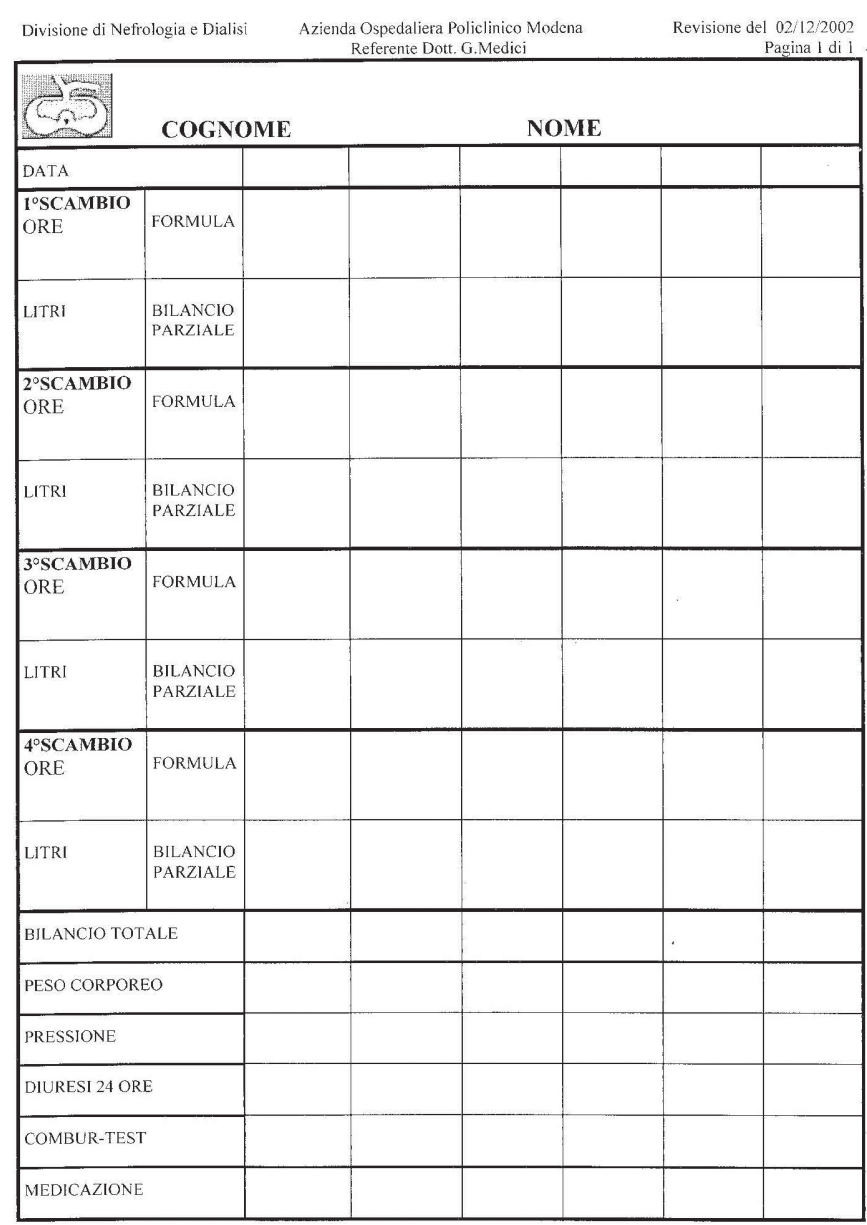

Fig. 1 - Esempio di scheda paziente. 
Divisione di Nefrologia e Dialis

Azienda Ospedaliera Políclinico Modena Referente Dott. G.Medici Pagina I di 1

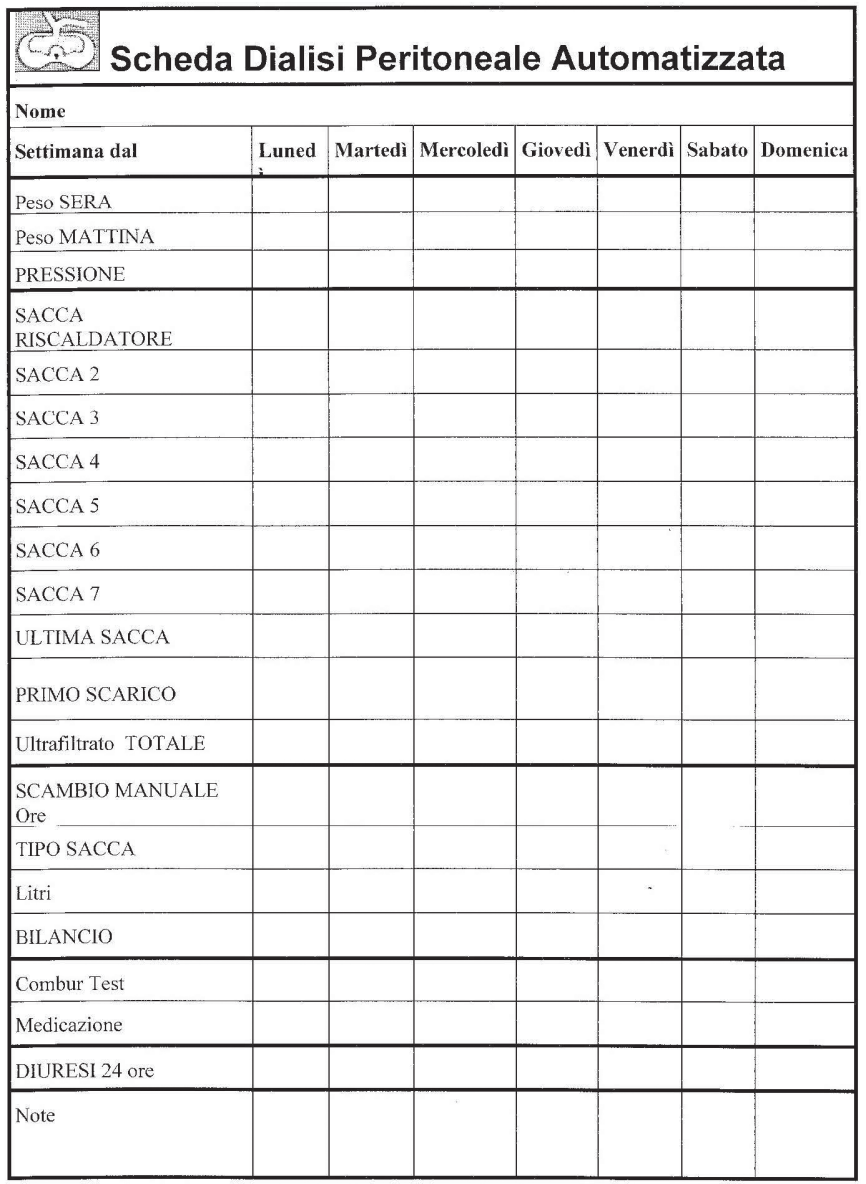

Fig. 2 - Scheda dialisi peritoneale automatizzata.

icodestrina, amminoacidi, ecc.), quantità del liquido di dialisi, tipo di trattamento (APD, CAPD) e frequenza settimanale (7 giorni a settimana o variabile in base alla funzione renale residua).

Nell'APD, invece, si utilizza uno schema caratterizzato da: tipo di formula (glucosio all'1,36\%, ecc.), volume di ogni singola sacca, numero di sacche, volume infuso per ciclo, tempo di sosta, numero di cicli e frequenza settimanale (Fig. 2). Anche nell'APD valgono le varianti della CAPD, a cui si aggiungono alcune specifiche particolarità legate alle caratteristiche tecniche della macchina e alla scelta di effettuare un trattamento terapeutico anche durante le ore diurne.

\section{Il test di equilibrazione peritoneale}

Il test di equilibrazione peritoneale (PET), nella versione classica e a oggi ancora utilizzata, proposta da Twardowski nel 1987 (2), è un procedimento che permette la valutazione semiquantitativa della funzione di trasporto della membrana peritoneale $(3,4)$. II trasporto di ciascun soluto viene determinato mediante la velocità di equilibrazione della concentrazione del soluto tra il plasma e il dialisato. II rapporto di concentrazione fra dialisato e plasma (D/P), in momenti specifici (determinati periodi di tempo $t$ ), indica il grado e la velocità di equilibrazione del soluto. Questo rapporto può essere determinato per diversi soluti che vengono trasportati dal sangue capillare al dialisato e viceversa. Quelli più comunemente analizzati per l'uso clinico sono: creatinina, urea, elettroliti, fosfati e proteine. Per quanto riguarda il glucosio, che, una volta trasferito dal dialisato al sangue viene velocemente metabolizzato, un valore di D/P sarebbe privo di significato (le concentrazioni di glucosio nel plasma rimangono sostanzialmente invariate durante il PET). La velocità di equilibrazione del glucosio viene quindi determinata dal rapporto tra la concentrazione di glucosio nel dialisato a orari specifici $(t)$ e la concentrazione di glucosio nella soluzione iniziale di dialisi indicata con Dt/DO $(4,5)$. II PET permette, inoltre, di valutare l'ultrafiltrazione peritoneale e l'eventuale presenza di liquido di dialisi residuo $(3,4)$. I valori ottenuti dal PET principalmente utilizzati riguardano il calcolo del $\mathrm{D} / \mathrm{P}$ della creatinina e il Dt/ D0 del glucosio. Grazie all'analisi di questi dati, Twardowski, nel suo test originale della durata di 4 ore, utilizzando una soluzione di dialisi contenente glucosio al 2,27\%, è stato in grado di tracciare delle curve di permeabilità della membrana peritoneale e, in base a queste, di classificare i pazienti in 4 grandi categorie:

- alti trasportatori ( $\mathrm{H}$ da High)

- medio-alti trasportatori (H-A da High-Average)

- medio-bassi traportatori (L-A da Low-Average)

- bassi trasportatori (L da Low)

Le 4 classi di trasportatori sono state ottenute sommando o sottraendo al valore medio di D/P creat e di D/DO una deviazione standard (DS). I pazienti con valori di D/P creat superiori alla media più la DS vengono classificati come $\mathrm{H}$; i pazienti con parametri compresi tra la media e la media più la DS vengono classificati come $\mathrm{H}$-A; i pazienti con valori compresi fra la media e la media meno la DS vengono classificati come L-A e, infine, i pazienti con valori inferiori alla media meno la DS vengono classificati come $L$.

Le ultime Best Practices della Società Italiana di Nefrologia hanno rivisto questa classificazione semplificandola, come viene descritto in seguito (5).

\section{La procedura per effettuare il PET}

La procedura standard per l'esecuzione del PET è stata proposta inizialmente da Twardowski, dal quale sono derivati molteplici altri test più complessi. Tuttavia recenti evidenze consigliano di introdurre alcune modifiche al test classico indicando la necessità di procedere a una nuova standardizzazione, in modo da ottenere dati il più possibile utili e riproducibili che consentano di confrontare i risultati sullo stesso paziente durante il periodo di terapia, valutazioni su differenti piani terapeutici nello stesso centro di dialisi e studi più 
ampi tra popolazioni di diverse aree geografiche $(3,4)$. Le Best Practices del Gruppo di Progetto di Dialisi Peritoneale infatti riportano come il test funzionale peritoneale di prima scelta dovrebbe essere il PET effettuato con soluzione di glucosio al $3,86 \%$, con una durata di 4 ore (PET modificato). Tale test deve includere la valutazione del sieving del sodio nel dialisato dopo 60 minuti dall'inizio del test ( $\triangle \mathrm{Na}$ a $\left.60^{\prime}\right)(5)$. II PET classico è standardizzato per i seguenti aspetti (3-4): 1) Durata dello scambio prima del test; 2) Volume di infusione e soluzione infusa; 3) Posizione del paziente durante l'infusione, la sosta e lo scarico; 4) Durata di infusione e di drenaggio; 5) Metodi di campionamento e conservazione; 6) Metodi di laboratorio.

\section{Modalità di esecuzione}

È necessario che il paziente arrivi al test con la cavità addominale piena (nei pazienti in CAPD effettuare il solito scambio notturno di 8 ore; nei pazienti in APD, se non fosse possibile effettuare uno scambio notturno di 8 ore, far terminare il programma notturno con un riempimento addominale; se anche ciò non fosse possibile, il paziente dovrebbe arrivare al Centro con la cavità addominale senza liquido da non oltre 45 minuti; nei pazienti con altri schemi dialitici peritoneali, effettuare lo scambio notturno come per i pazienti in CAPD).

- Fase 1: la sera prima dell'esame il paziente infonde la soluzione di dialisi all'1,36\% o al 2,27\%; la mattina dell'esame viene preparata una sacca da 2 litri di soluzione di dialisi al $4.25 \%$ di glucosio idrato (o al 3,86\% di glucosio anidro) e riscaldata fino a raggiungere la temperatura corporea;

- Fase 2: con il paziente in posizione eretta (o seduta) effettuare il drenaggio del dialisato per 20-30' della sosta prima del PET, raccogliendolo in un recipiente;

- Fase 3: con il paziente supino si procede all'infusione della soluzione, precedentemente riscaldata, a una velocità di 400 $\mathrm{mL}$ ogni 2'; vengono impiegati esattamente 10' per effettuare l'intero carico di 2 litri (solo se il paziente non la tollera verrà infusa una quantità minore); contemporaneamente, occorre far ruotare il paziente da un fianco all'altro dopo ogni infusione da $400 \mathrm{~mL}$; l'ora esatta in cui viene completata l'infusione è il tempo 0 da ci misurare l'ora di stasi;

- Fase 4: al tempo 0 occorre prelevare i campioni di dialisato secondo le seguenti modalità: far ruotare il paziente da un fianco all'altro per 2 volte, collegare una siringa da 30 o $50 \mathrm{~mL}$ al tubo di scarico, aspirare $30 \mathrm{~mL}$ (per lo spazio morto di linee e catetere) e scartare, bloccare il drenaggio, collegare una siringa da $20 \mathrm{~mL}$ e aspirare completamente ( $10 \mathrm{~mL}$ per gli esami, $10 \mathrm{~mL}$ di riserva);

- Fase 5: dopo 2 ore di stasi prelevare i campioni di dialisato seguendo la stessa procedura precedentemente descritta nella Fase 4; alla seconda ora viene eseguito il prelievo ematico;

- Fase 6: dopo 4 ore di stasi, in cui il paziente può muoversi liberamente, si esegue il drenaggio completo con il paziente in posizione seduta; senza effettuare il lavaggio della linea, mescolare il liquido e prelevare l'ultimo campione come da Fase 4.

I dati ottenibili dal test sono:

- D0: Glucosio nel liquido di dialisi al tempo 0 in $\mathrm{mg} / \mathrm{dL}$;

- D4: Glucosio nel liquido a 4 ore in $\mathrm{mg} / \mathrm{dL}$;

- P creat: Creatininemia a 2 ore;

- D creat: Creatinina nel liquido a 2 ore $\mathrm{mg} / \mathrm{dL}$;

- D creat: Creatinina nel liquido a 4 ore $\mathrm{mg} / \mathrm{dL}$.

Per l'interferenza delle elevate concentrazioni di glucosio in alcuni metodi di dosaggio delle concentrazioni di creatinina nel liquido di dialisi viene utilizzato un fattore di correzione (specifico per ogni laboratorio). Di conseguenza vengono calcolati:

- D creat corretta a 2 ore: Creatinina nel liquido corretta a 2 ore;

- D creat corretta a 4 ore: Creatinina nel liquido corretta a 4 ore;

- D2/D0 glucosio a 2 ore;

- D4/D0 glucosio a 4 ore;

- D/P creatinina corretta a 2 ore;

- $\mathrm{D} / \mathrm{P}$ creatinina corretta a 4 ore.

\section{I risultati del PET}

Secondo quanto riportato nelle Best Practices del Gruppo di Progetto di Dialisi Peritoneale (5), i pazienti dovrebbero essere classificati in base ai risultati del 3,86\%-PET:

1. In base ai valori di ultrafiltrazione in pazienti con normale ultrafiltrazione $(\geq 400 \mathrm{~mL})$ e con perdita della capacità di ultrafiltrazione $(<400 \mathrm{~mL})$.

2. In base ai valori di D/P creat in Rapidi (Alti), Medio alti e Medio bassi (Medi) e Lenti (Bassi) trasportatori.

Valori di D/P creat $>0,80$ indicano i pazienti Rapidi (Alti) trasportatori.

Valori di $\mathrm{D} / \mathrm{P}$ creat $<0,60$ indicano i pazienti Lenti (Bassi) trasportatori.

Valori di $D / P$ creat compresi fra 0,60 e 0,80 indicano $i$ Medi trasportatori.

3. In base ai valori di $\Delta \mathrm{Na}$ a 60 minuti in pazienti con sieving del $\mathrm{Na}$ conservato $(\Delta \mathrm{Na} \geq 5 \mathrm{mmol} / \mathrm{L})$ e in pazienti con ridotto sieving del $\mathrm{Na}(\Delta \mathrm{Na}<5 \mathrm{mmol} / \mathrm{L})$.

I risultati del test dovrebbero essere utilizzati per la prescrizione e per l'ottimizzazione della terapia dialitica peritoneale.

Per i pazienti Rapidi (Alti) trasportatori è indicato il trattamento dialitico peritoneale automatizzato (APD) e, se necessario, con la sosta lunga effettuata con l'icodestrina.

Per i pazienti Lenti (Bassi) trasportatori è indicato il trattamento dialitico peritoneale manuale (CAPD) con volumi tendenzialmente elevati del singolo scambio (High-Dose 
CAPD). Nel caso tali volumi non fossero tollerati o si verificasse comunque un'inadeguatezza dialitica e/o ultrafiltrativa, va preso in considerazione il passaggio all'emodialisi.

Una riduzione marcata del sieving del $\mathrm{Na}$ a 60 minuti $(\Delta \mathrm{Na}$ $<5 \mathrm{mmol} / \mathrm{L}$ ) indica una riduzione del trasporto di acqua libera peritoneale e, spesso, l'aumento dell'osmolarità (concentrazione di glucosio) della soluzione non determina un aumento dell'ultrafiltrazione. Ciò può essere confermato dalla quantificazione del trasporto di acqua libera. In tal caso, per aumentare l'ultrafiltrazione, utilizzare l'icodestrina. Utile la ripetizione a breve ( 3 mesi) del PET e, in caso di perdita ulteriore di ultrafiltrazione e/o $\Delta \mathrm{Na}$, prendere in considerazione il passaggio all'emodialisi.

\section{Problemi nutrizionali nel paziente in dialisi peritoneale}

Uno dei principali problemi correlati alla dialisi peritoneale (DP) riguarda il mantenimento del paziente in uno stato nutrizionale ottimale. È noto che l'insufficienza renale cronica e il trattamento dialitico favoriscono la comparsa di numerose alterazioni metaboliche. In questi pazienti è possibile la comparsa di malnutrizione proteica (6), in quanto la tecnica dialitica determina una perdita di aminoacidi nel liquido di dialisi che, non adeguatamente corretta, può peggiorare un quadro di malnutrizione proteica preesistente o portare all'instaurarsi di una nuova condizione. Sotto il profilo lipidico, i pazienti trattati con DP tendono ad avere livelli elevati di trigliceridi, di colesterolo totale e di colesterolo LDL e bassi valori di HDL e a sviluppare di conseguenza un ambiente pro-aterogeno (7). Sotto l'aspetto glucidico si può riscontrare la comparsa di iperinsulinemia, iperglicemia e insulino-resistenza (8), con conseguente predisposizione all'intolleranza glucidica, al diabete o all'obesità $(6,8)$. Recenti studi hanno dimostrato l'aumento di rischio di mortalità dei pazienti sottoposti a DP con BMI (Body Mass Index) superiore a 30, rispetto agli omologhi trattati con emodialisi (9-11). Si evidenzia quindi la necessità di monitorare costantemente un'eventuale malnutrizione per eccesso nei pazienti sottoposti a questo tipo di trattamento sostitutivo. Allo stesso modo può comparire una malnutrizione per difetto dovuta a fattori legati a eventuali malattie associate (malattie cardio-vascolari, ecc.), allo stress ossidativo oppure a condizioni di ridotta assunzione di alimenti, senso di sazietà precoce, difficoltà nella gestione dei pasti, solitudine, depressione, anoressia, disgeusia e così via (12). Dal momento che la deplezione proteico-energetica rappresenta uno dei principali determinanti, insieme alla comorbidità cardiovascolare, dell'elevata mortalità dei pazienti in dialisi (13), è necessario identificare il fabbisogno energetico adeguato, definire l'energia introdotta con le sacche di dialisi, stabilire un corretto fabbisogno proteico e raggiungere i fabbisogni di fosforo, potassio, sodio e liquidi, secondo le raccomandazioni.

\section{Fabbisogno energetico}

Le Linee Guida internazionali (KDOQI, Kidney Disease Outcomes Quality Initiative; ESPEN, European Society for Clinical Nutrition and Metabolism; EBPG, European Renal Best Practice; EDTNA/ERCA, European Dialysis and Transplant Nurses Association/European Renal Care Association) indicano che un corretto apporto energetico deve fornire tra le 30 e le $35 \mathrm{Kcal}$ per kg di peso al giorno (Tab. I) (14-17).

\section{Fabbisogno proteico}

In corso di DP le perdite di proteine possono arrivare fino a $20 \mathrm{~g}$ al giorno. Le indicazioni delle diverse società scientifiche dell'ambito nefrologico, sia europee che internazionali, raccomandano un introito proteico da 1 a 1,5 g/ kg di peso al giorno per permettere un bilancio azotato positivo o in pareggio. II $50 \%$ dovrebbe essere costituito da proteine a elevato valore biologico (HBW) per facilitare il loro uso da parte dell'organismo e favorire un adeguato apporto di amminoacidi essenziali (Tab. II).

TABELLA I - Fabbisogno energetico suggerito per pazienti in dialisi peritoneale

\begin{tabular}{|c|c|c|c|c|}
\hline & KDOQI & ESPEN & EBPG & EDTNA/ERCA \\
\hline Energia & $\begin{array}{l}25-35 \mathrm{kcal} / \mathrm{kg} \text { BW/die } \\
\text { in base a età, sesso, livello di attività fisica } \\
\text { composizione corporea, obiettivi di peso, } \\
\text { stadio di insufficienza renale cronica e } \\
\text { malattia concomitante o presenza di } \\
\text { infiammazione per mantenere il normale } \\
\text { stato nutrizionale }\end{array}$ & $\begin{array}{l}35 \mathrm{kcal} / \mathrm{kg} \text { IBW/die } \\
\text { lipidi } 30-40 \% \text { energia totale; } \\
\text { carboidrati complessi } 25-40 \% \text {; } \\
\text { oligosaccaridi limitati. } \\
\text { L'assorbimento del glucosio deve } \\
\text { essere preso in considerazione }\end{array}$ & - & $\begin{array}{l}35 \mathrm{kcal} / \mathrm{kg} \text { IBW/die } \\
30 \mathrm{kcal} / \mathrm{kg} / \mathrm{IBW} \text { die negli } \\
\text { anziani e nei pazienti con } \\
\text { attività ridotta. } \\
\text { Includere le calorie } \\
\text { dall'assorbimento } \\
\text { peritoneale del glucosio. }\end{array}$ \\
\hline
\end{tabular}

Abbreviazioni: $\mathrm{BW}=$ peso corporeo; $\mathrm{IBW}$ = peso corporeo ideale.

TABELLA II - Fabbisogno proteico suggerito per pazienti in dialisi peritoneale

\begin{tabular}{|c|c|c|c|c|}
\hline & KDOQI & ESPEN & EBPG & EDTNA/ERCA \\
\hline Proteine & $\begin{array}{l}\text { 1,0-1,2 g/kg BW/die } \\
\text { per mantenere uno stato } \\
\text { nutrizionale stabile }\end{array}$ & $\begin{array}{l}\text { 1,2-1,5 g/kg IBW/die } \\
\geq 50 \% \mathrm{HBV} \\
\text { maggiore assunzione in caso } \\
\text { di peritonite }\end{array}$ & - & $\begin{array}{l}\text { 1,0-1,2 g/kg IBW/die } \\
\geq 50 \% \mathrm{HBV} \\
1,5 \mathrm{~g} / \mathrm{kg} \text { IBW/die } \\
\text { in caso di peritonite }\end{array}$ \\
\hline
\end{tabular}

Abbreviazioni: $\mathrm{BW}=$ peso corporeo; IBW = peso corporeo ideale. 
TABELLA III - Fabbisogno di fosforo, potassio, sodio e liquidi suggerito per pazienti in dialisi peritoneale

\begin{tabular}{|c|c|c|c|c|}
\hline & KDOQI & ESPEN & EBPG & EDTNA/ERCA \\
\hline Fosforo & $\begin{array}{l}\text { si raccomanda di regolare l'assunzione di fosforo nella } \\
\text { dieta per mantenere i livelli sierici di fosfato nell'intervallo } \\
\text { normale }\end{array}$ & $17 \mathrm{mg} / \mathrm{kg} / \mathrm{IBW}$ & - & $1000-1400 \mathrm{mg} / \mathrm{die}$ \\
\hline Potassio & $\begin{array}{l}\text { è ragionevole regolare l'assunzione di potassio nella dieta } \\
\text { per mantenere la potassiemia nel range di normalità. }\end{array}$ & $<1 \mathrm{mEq} / \mathrm{kg} / \mathrm{die}$ & - & 2000-2500 mg/die \\
\hline Sodio & $100 \mathrm{mmol} / \mathrm{die}$ (o <2,3 g/die) & $60-100 \mathrm{mEq} / \mathrm{die}$ & - & $1800-2500 \mathrm{mg} / \mathrm{die}$ \\
\hline Liquidi & N/A & $\begin{array}{l}500-800 \mathrm{ml} / \mathrm{die}+\text { volume } \\
\text { urinario residuo (tenendo } \\
\text { conto del contenuto di } \\
\text { liquidi degli alimenti) }\end{array}$ & - & $\begin{array}{l}800 \mathrm{ml}+\text { produzione giornaliera } \\
\text { di urina - includere solo cibi } \\
\text { liquidi a temperatura ambiente } \\
\text { e quelli con un alto contenuto } \\
\text { di liquidi }\end{array}$ \\
\hline
\end{tabular}

Abbreviazioni: BW = peso corporeo; IBW = peso corporeo ideale

\section{Fabbisogno di fosforo, potassio, sodio e liquidi}

Per quanto concerne l'assunzione di elettroliti e liquidi, le Linee Guida indicano quanto riportato nello schema sottostante (Tab. III).

\section{Calcolo delle calorie introdotte con le sacche di dialisi}

Per determinare le richieste energetiche dei pazienti in DP è necessario quantificare il glucosio assorbito dal dialisato per ciascun paziente. Sono stati presi in considerazione due metodi per stimare l'assorbimento del glucosio: la formula di Grodstein et al. (18) e la formula D/D0 (19).

\section{La Grodstein et al. formula}

Lo studio di Grodstein et al., pubblicato sul Kidney International vol. 3 dell'anno 1981, è stato uno dei primi studi a occuparsi della quantificazione dell'assorbimento del glucosio in CAPD. Diciannove studi della durata compresa tra i 4 e i 21 giorni vennero effettuati in 7 uomini uremici cronici, di età compresa tra i 27 e i 59 anni, clinicamente stabili, sottoposti a CAPD. I pazienti avevano iniziato il trattamento dialitico peritoneale intermittente e/o CAPD dai 6 ai 50 mesi precedenti. Durante ogni studio i pazienti ebbero un regime fisso di CAPD con numero, durata degli scambi e concentrazione di glucosio del dialisato costanti. Vennero loro assegnate diete costanti che fornivano $32,9 \pm 3,5 \mathrm{kcal} / \mathrm{kg}$ di peso corporeo al giorno e $1,0 \pm 0,11$ oppure $1,4 \pm 0,08 \mathrm{~g}$ di proteine per $\mathrm{kg}$ di peso corporeo al giorno. Dalle 3 alle 5 sacche da 2 litri di soluzioni di dialisi vennero infuse ogni giorno nella cavità peritoneale con il metodo di Oreopoulos et al.; il numero di scambi venne regolato in ogni paziente per ottenere la clearance totale dell'urea. Due studi vennero condotti con 3 scambi/giorno, sei studi con 4 scambi/giorno e undici studi con 5 scambi/ giorno. Il numero degli scambi con sacche da $1,5 \mathrm{~g} / \mathrm{dL}$ o da $4,25 \mathrm{~g} / \mathrm{dL}$ di destrosio venne prescritto in base alla necessità di rimozione del liquido in ogni paziente. Ogni mattina, dopo un digiuno di 8 ore, venne effettuata la misurazione del glucosio plasmatico. Inoltre, vennero effettuate determinazioni di glucosio sia nel dialisato infuso che nel totale del dialisato effluito. Più volte venne controllata la concentrazione del glucosio nelle sacche del liquido di dialisi. Prima di ogni scambio e dopo, il liquido di afflusso e di deflusso venne pesato e i volumi vennero calcolati utilizzando il valore del peso e del peso specifico delle 16 soluzioni. I volumi misurati sulle sacche da 2 litri di dialisato di afflusso furono 2,05 $\pm 0,008 \mathrm{~L}$ per la soluzione all' $1,5 \%$ e 2,06 $\pm 0,012$ L per quella al 4,25\%. L'assorbimento del glucosio giornaliero venne quindi calcolato come la differenza tra la quantità di glucosio instillata nella cavità peritoneale e l'importo drenato ogni giorno. La concentrazione media di glucosio (espressa in $\mathrm{g} / \mathrm{dL}$ ) del dialisato infuso, per ogni giorno, venne definita come la quantità totale di glucosio diviso per il volume totale infuso. Per il calcolo dell'energia derivata dal dialisato venne considerata l'uguaglianza tra $1 \mathrm{~g}$ di glucosio assorbito e 3,7 kcal/g. I risultati permisero a Grodstein et al. di notare una forte correlazione tra la quantità di glucosio assorbito giornalmente e la quantità di glucosio instillato.

Questo consenti di definire una formula che a tutt'oggi consente di calcolare la quantità di glucosio assorbito per ogni litro di dialisato infuso. La Grodstein et al. Formula (18) è la seguente: $Y=(11,3 X-10,9)$, dove: $Y=$ glucosio assorbito per litro di dialisato $(\mathrm{g} / \mathrm{L})$ e $\mathrm{X}=$ concentrazione media del glucosio nel dialisato ( $\mathrm{g} / \mathrm{dL}$ ) al giorno; da questa, per ottenere il glucosio assorbito giornalmente, basta moltiplicare il valore di $Y$ ottenuto per i litri di dialisato infuso nelle 24 ore.

Esempio di calcolo: un paziente in CAPD che esegue uno schema di dialisi con 5 scambi al giorno di cui 2 scambi con 2 litri di dialisato all' $1,5 \%$ (destrosio $1,30 \mathrm{~g} / \mathrm{dL}$ ) e 3 scambi con 2 litri al 4,25\% (destrosio 3,76 g/dL) ha una concentrazione media giornaliera di destrosio di 2,80 g/dL. La quantità di glucosio assorbita ottenuta con la formula è: $Y=(11,3 \times 2,8)-$ 10,9 , quindi $Y=20,7 \mathrm{~g} / \mathrm{L}$. Dato che la quantità di soluzione infusa giornalmente è di 10 litri, il glucosio assorbito nelle 
24 ore dal liquido di dialisi sarà pari a: glucosio 24 ore $=20,7 \times 10$, vale a dire glucosio 24 ore $=207 \mathrm{~g}$.

\section{La D/DO formula}

Partendo dai dati dell'indagine PET, Bodnar DM et al. nel 1993 hanno proposto l'utilizzo del D/D0 nella determinazione dell'assorbimento del glucosio (19). La formula che utilizza il D/D0 ottenibile dal test di equilibrazione peritoneale (PET) proposta da Bodnar DM et al. è la seguente: assorbimento di glucosio 24 ore $=(1-D / D 0) x j$, dove: $x j=$ quantità totale di glucosio infuso in g e D/DO = coefficiente ottenuto dal PET. In base alla metodica di dialisi peritoneale, il D/D0 può essere quello alla quarta ora, nel caso in cui il paziente sia trattato in modalità CAPD, o quello del tempo di sosta, se trattato in APD. Dal momento che i dati del PET sono di tipo semiquantitativo, i valori ottenuti sono una stima e non un valore assoluto dell'assorbimento del glucosio.

Esempio di calcolo: utilizzando i dati del paziente precedentemente trattato nell'esempio della Grodstein et al. formula (13) e ipotizzando un coefficiente di $\mathrm{D} 4 / \mathrm{D0}=0,58$, otteniamo la seguente stima: glucosio 24 ore $=(1-0,58) \times$ $(2,8 \times 10 \times 10)=117,6 \mathrm{~g}$, dove: $x \mathrm{j}=$ quantità media di glucosio infuso $(\mathrm{g} / \mathrm{dL}) \times$ fattore di conversione da decilitro a litro $x$ litri di dialisato totale infuso.

\section{Grodstein et al. formula vs D/D0 formula}

Dall'analisi delle formule risulta evidente come si ottengano risultati diversi. Uno studio comparativo tra le due formule di calcolo e l'assorbimento reale di glucosio nelle 24 ore (glucosio instillato in entrata - glucosio rimanente nel liquido di dialisi in uscita) è stato realizzato da Bodnar DM et al. (19). L'assorbimento di glucosio nelle 24 ore è stato misurato per 50 volte in 40 pazienti in CAPD e in 17 pazienti in APD. Una volta ottenuti i dati del glucosio reale assorbito, sono stati valutati gli errori delle due formule precedenti comparandoli tramite il Wilconxon signed rank test (19). I risultati dello studio hanno dimostrato che, nonostante nessuna delle due formule calcolasse perfettamente l'assorbimento di glucosio assoluto, la formula D/DO è molto più vicina al vero valore, rispetto alla Grodstein et al. formula. In generale la D/DO formula è risultata più precisa per tutti i tipi di trasportatori e in particolare fornisce dati che più si avvicinano alla realtà negli Alti e Bassi trasportatori, per i quali si verifica il massimo grado di errore utilizzando la Grodstein et al. formula.

Nello studio compare anche un confronto tra la D/DO formula e la Grodstein et al. formula in pazienti in APD. Nonostante la Grodstein et al. non sia stata formulata e proposta per questo tipo di pazienti che sono sottoposti a tempi di sosta più brevi, si evidenzia come la D/DO formula risulti più accurata nella stima dell'assorbimento del glucosio anche in questi casi (19).

\section{Esempio di calcolo delle formule su pazienti in CAPD: la nostra esperienza}

Utilizzando i dati di 3 pazienti in CAPD che hanno eseguito il PET secondo lo standard dell'Unità Operativa, ipotizzando 2 tipi di schemi di dialisi differenti, abbiamo calcolato i grammi di glucosio assorbiti utilizzando la D/DO formula e la Grodstein et al. formula.

Lo schema di dialisi A prevede una infusione giornaliera di:

- $8 \mathrm{~L}$ di soluzione di dialisi all'1,36\%; mentre lo schema di dialisi B prevede una infusione giornaliera di:

- $4 \mathrm{~L}$ di soluzione di dialisi all'1,36\% e $4 \mathrm{~L}$ di soluzione di dialisi al 2,27\%.

Per i calcoli sono state utilizzate le formule precedentemente illustrate.

La Tabella IV mostra i risultati ottenuti, in termini di assorbimento di glucosio, a seconda dello schema di dialisi e della formula utilizzati.

TABELLA IV - Esempio di calcolo in pazienti in capd che hanno eseguito il pet

\begin{tabular}{|c|c|c|c|c|c|c|c|c|c|}
\hline & & & & & & $\begin{array}{l}\text { Schema } \\
\text { dialisi A }\end{array}$ & $\begin{array}{l}\text { Schema } \\
\text { dialisi B }\end{array}$ & $\begin{array}{l}\text { Schema } \\
\text { dialisi A }\end{array}$ & $\begin{array}{l}\text { Schema } \\
\text { dialisi B }\end{array}$ \\
\hline & & & & & & $\begin{array}{l}D / D_{0} \\
\text { formula }\end{array}$ & $\begin{array}{l}D / D_{0} \\
\text { formula }\end{array}$ & $\begin{array}{l}\text { Grodstein } \\
\text { formula }\end{array}$ & $\begin{array}{l}\text { Grodstein } \\
\text { formula }\end{array}$ \\
\hline Paziente & Età & Sesso & $\begin{array}{c}\text { Tempo } \\
\text { PET }\end{array}$ & $\begin{array}{l}\text { Tipo } \\
\text { trasp. }\end{array}$ & $\begin{array}{c}D / D_{0} 4 \\
\text { ore }\end{array}$ & $\begin{array}{c}\text { Glucosio } \\
\text { (g/die) }\end{array}$ & $\begin{array}{c}\text { Glucosio } \\
\text { (g/die) }\end{array}$ & $\begin{array}{c}\text { Glucosio } \\
\text { (g/die) }\end{array}$ & $\begin{array}{c}\text { Glucosio } \\
\text { (g/die) }\end{array}$ \\
\hline BS & 61 & $\mathrm{~F}$ & 6 sett. & $\begin{array}{l}\text { MEDIO- } \\
\text { ALTO }\end{array}$ & 0,35 & 71 & 94 & 36 & 77 \\
\hline$C R$ & 61 & $\mathrm{M}$ & 8 sett. & $\begin{array}{l}\text { MEDIO- } \\
\text { ALTO }\end{array}$ & 0,30 & 76 & 102 & 36 & 77 \\
\hline SM & 77 & M & 6 sett. & $\begin{array}{l}\text { MEDIO- } \\
\text { BASSO }\end{array}$ & 0,38 & 67 & 90 & 36 & 77 \\
\hline
\end{tabular}




\section{Conclusioni}

I risultati ottenuti da entrambe le formule possono essere valutati singolarmente oppure essere messi a confronto; non essendo, però, né valori assoluti, né costanti nel tempo, né sufficienti, da soli, a definire il fabbisogno energetico, è necessario che siano sempre valutati in modo critico. Tuttavia, studiare l'assorbimento del glucosio in modo sistematico in tutti i pazienti in DP, monitorando periodicamente nel tempo le variazioni dell'assorbimento del glucosio stesso in relazione alle modificazioni della membrana peritoneale in corso di DP, potrebbe contribuire in modo significativo ad adeguare il trattamento nutrizionale alle esigenze dei pazienti in DP, in particolare per quanto riguarda la definizione dell'apporto energetico.

\section{Disclosures}

Conflict of interest: The authors declare no conflict of interest. Financial support: This research received no specific grant from any funding agency in the public, commercial, or not-for-profit sectors.

Authors contribution: All authors contributed equally to this manuscript.

\section{Bibliografia}

1. Amici G. La prescrizione dialitica in dialisi peritoneale.. Best Practice. Gruppo di Studio Dialisi Peritoneale, Società Italiana di Nefrologia. 2012 Online (accesso Dicembre 2021)

2. Twardowski ZJ, Nolph KD, Khanna R, et al. Peritoneal Equilibration test. Perit Dial Int. 1987;7(3):138-148. CrossRef

3. V. La Milia, Test di equilibrazione peritoneale: attualità e prospettive future. G Ital Nefrol 2007;24(6):510-525. Online (Accesso Dicembre 2021)

4. Misra M, Khanna R. Peritoneal equilibration test. Uptodate. 2012. Online (Accesso Dicembre 2021)

5. La Milia V. Valutazione funzionale della membrana peritoneale. Best Practice Società Italiana di Nefrologia. 2010. Online (Accesso Dicembre 2021)

6. Mattei R. Manuale di nutrizione clinica. 2006, Milano: Franco Angeli Editore, Collana Medi-care. 2006:247-8.
7. Ganesh SK, Hulbert-Shearon T, Port FK, Eagle K, Stack AG. Mortality differences by dialysis modality among incident ESRD patients with and without coronary artery disease. J Am Soc Nephrol. 2003;14(2):415-424. CrossRef PubMed

8. Burkart J. Metabolic consequences of peritoneal dialysis. Semin Dial. 2004;17(6):498-504. CrossRef PubMed

9. Stack AG, Murthy BV, Molony DA. Survival differences between peritoneal dialysis and hemodialysis among "large" ESRD patients in the United States. Kidney Int. 2004;65(6):2398-2408. CrossRef PubMed

10. Abbott KC, Glanton CW, Trespalacios FC, et al. Body mass index, dialysis modality, and survival: analysis of the United States Renal Data System Dialysis Morbidity and Mortality Wave II Study. Kidney Int. 2004;65(2):597-605. CrossRef PubMed

11. McDonald SP, Collins JF, Johnson DW. Obesity is associated with worse peritoneal dialysis outcomes in the Australia and New Zealand patient populations. J Am Soc Nephrol. 2003;14(11):2894-2901. CrossRef PubMed

12. Pasticci F, Fantuzzi AL, Pegoraro M, McCann M, Bedogni G. Nutritional management of stage 5 chronic kidney disease. J Ren Care. 2012;38(1):50-58. CrossRef PubMed

13. A. Cupisti e al. La terapia dietetica nutrizionale nella gestione del paziente con Malattia Renale Cronica in fase avanzata per ritardare l'inizio e ridurre la frequenza della dialisi, e per il programma di trapianto pre-emptive. Consensus document. $G$ Ital Nefrol. 2018. Online (Accesso Dicembre 2021)

14. Ikizler TA, Burrowes JD, Byham-Gray LD, et al; KDOQI Nutrition in CKD Guideline Work Group. KDOQI clinical practice guideline for nutrition in CKD: 2020 update. Am J Kidney Dis. 2020;76(3) (suppl 1):S1-S107. CrossRef PubMed

15. N.J.M. Cano a,b,c, M. Aparicio d, G. Brunori e, J.J. Carrero f, B. Cianciaruso g, E.Fiaccadori h, B. Lindholm f, V. Teplan i, D. Fouque j, G. Guarnieri k ESPEN Guidelines on Parenteral Nutrition: Adult Renal Failure. J Clin Nutr. 2009;28:401-414.

16. Fouque D, Vennegoor M, Piet Ter Wee et al. EBPG Guideline on Nutrition, Nephrology Dialysis Transplantation, Volume 22, Issue suppl_2, May 2007, Pages ii45-ii87, CrossRef

17. European Guidelines for Nutritional care of Adult Renal Patients. Journal of Renal Care 2003;29(1):223-43. CrossRef

18. Grodstein GP, Blumenkrantz MJ, Kopple JD, Moran JK, Coburn JW. Glucose absorption during continuous ambulatory peritoneal dialysis. Kidney Int. 1981;19(4):564-567. CrossRef PubMed

19. Bodnar DM, Busch S, Fuchs J, Piedmonte M, Schreiber M. Estimating glucose absorption in peritoneal dialysis using peritoneal equilibration tests. Adv Perit Dial. 1993;9:114-118. PubMed 